\title{
Genetic association and path coefficient analysis among yield and nutritional traits of tomato (Lycopersicon esculentum $\mathrm{L}$.)
}

\author{
Shaila Sharmin ${ }^{1}$, Afsana Hannan ${ }^{1}$, Md Tahjib-Ul-Arif ${ }^{2}$ and ${ }^{\otimes}$ G H M Sagor ${ }^{1}$ \\ ${ }^{1}$ Department of Genetics and Plant Breeding, Bangladesh Agricultural University, Mymensingh-2202, Bangladesh \\ ${ }^{2}$ Department of Biochemistry and Molecular Biology, Bangladesh Agricultural University, Mymensingh-2202, Bangladesh
}

\begin{tabular}{|c|c|}
\hline ARTICLE INFO OPENC & $\mathbf{t}$ \\
\hline $\begin{array}{l}\text { eceived : } 05 \text { March } 2019 \\
\text { ccepted : } 29 \text { May } 2019 \\
\text { blished: } 30 \text { June } 2019 \\
\end{array}$ & \multirow{3}{*}{$\begin{array}{l}\text { The present study was conducted following randomized complete block design with three replications to } \\
\text { evaluate the genetic variability of twenty five tomato genotypes for yield and nutritional traits and also } \\
\text { their attributing factors. A wide range of variation was observed among the characters studied which have } \\
\text { a great interest for tomato breeders. Heritability in broad and narrow sense for soluble solid in green and } \\
\text { red tomato, total phenolic content, fruit diameter and seed/fruit was } 93.23 \%, 98.58 \%, 99.92 \%, 99.37 \% \text { and } \\
96.15 \% \text { respectively and for those traits, phenotypic influence was negligible. Leaf chlorophyll content } \\
\text { and total phenolic content showed positive significant correlation with soluble solid (sugar) whereas } \mathrm{p}^{\mathrm{H}} \\
\text { showed negative correlation. Yield/plant was found highly significant and positively correlated with } \\
\text { individual fruit weight, fruit diameter, seed/fruit, and plant height whereas soluble solid, leaf chlorophyll } \\
\text { content, total phenolic content showed negative correlation. Path analysis revealed that soluble solid had } \\
\text { positive direct effect with leaf chlorophyll content, } \mathrm{p}^{\mathrm{H}} \text { of fruit juice and days to first flowering and } \\
\text { negative direct effects with individual fruit weight, fruit diameter, plant height, fruit /bunch, whereas } \\
\text { yield/plant showed positive direct effects with all above traits except soluble solids in red tomato, which } \\
\text { clearly indicate inverse correlation between yield and nutritional components of tomato fruit. Further, } \\
\text { principal component analysis found that four principal components contributed } 75.1 \% \text { of the total } \\
\text { variability. Individual fruit weight, days to first flowering, pH of fruit juice, fruit/bunch and soluble solids } \\
\text { in fruits were found to be the most important traits in PC1, PC2, PC3, PC4 and PC5, respectively. As } \\
\text { soluble solid content, yield and its component traits have high heritability, therefore, improvement is also } \\
\text { possible using breeding approaches. }\end{array}$} \\
\hline Components & \\
\hline & \\
\hline
\end{tabular}

(92019 by authors and BAURES. This work is licensed under the Creative Commons Attribution International License (CC By 4.0).

\section{Introduction}

Tomato (Lycopersicon esculentum L.) is one of the most important and popular vegetables in the world. It belongs to the family Solanaceae with chromosome number $2 \mathrm{n}=24$ with small genome size $(950 \mathrm{Mb}$ per haploid nucleus) (Jenkins, 1948) and is normally a self pollinated annual crop. Cultivated tomato is the second most commonly consumed vegetable, just next to potato. Many developing countries like Bangladesh benefited from the green revolution in cereal production in the past but were not able to substantially reduce poverty and malnutrition. Vegetable production can help farmers generate income which eventually alleviate poverty. At present $6.10 \%$ cultivable land area $(48,538$ acres $)$ is under tomato cultivation both in winter and summer (BBS, 2008). It is cultivated all over the country due to its adaptability to wide range of soil and climate (Ahmed, 1976). The estimated annual production of tomato in Bangladesh was 368121 metric tonnes in 2015-2016 fiscal years (BBS, 2016). To meet up local demand, Bangladesh government imported 10935 metric tonnes from foreign countries in the same time (BBS, 2016). Now-a-days tomato is very popular not only to the consumers for its health benefits but also to the Cite this article

Sharmin, S., Hannan, A., Arif M.T.U. and Sagor, G H M. 2019. Genetic association and path coefficient analysis among yield and nutritional traits of tomato (Lycopersicon esculentum L.). Journal of Bangladesh Agricultural University, 17(1): 187-193. https://doi.org/10.3329/jbau.v17i2.41942 farmers for its high market value and as well as researcher for its genetics and genomic characters. The tomato is classified as a functional food, for having good levels of vitamins, minerals, and especially lycopene, a carotenoid pigment that provides red color and has antioxidant qualities (Alvarenga, 2004) which acts as an anticarcinogen (Bhutani and Kallo, 1983). To meet the increasing demand of tomato, it is important to study the genetic variability of tomato as variability assessment among tomato genotypes helps to maintain and utilize germplasm resources for the improvement of the cultivars (Reddy et al., 2013). Morphological traits play a vital role in determining the important characters, variability and genetic relationship among the genotypes (Osei et al., 2014). Tomato fruit yield is the final result which is associated with other yield contributing traits and theses traits again interrelated among them (Islam and Khan, 1991). Besides yield, quality of fresh market tomato is also important and it (Lycopersicon esculentum Mill.) is affected by fruit appearance, flavor (taste and aroma) (Shewfelt, 1993) and texture (Causse et al., 2001; Vickers, 1977). Although the perception of flavor is influenced by many factors, taste (sweetness, sourness) is one of its most important components and it 
is determined basically by sugars and acids (Kader et al., 1977; Malundo et al., 1995; Stevens et al., 1977a, b). Sugar, organic acids, phenols and minerals are the main constituents of tomato taste, among them sugars quantitatively making the largest contribution (Kader, 2008). Generally reducing sugars correlate with soluble solids content, hence soluble solids measurements provides a good estimate of the sugar level. (Kader et al., 1977). Many cultivars were selected for traits such as resistance to stresses, uniformity, appearance, firmness, extended shelf life, in contrast to the desire of consumers for sweet tomatoes (Shwefelt, 2000). Reinforcement of the breeding strategy also comes from the fact that consumers appear conflicted in their desires; while taste is given high importance, fruit of poor appearance will not be chosen even if the taste can't be guaranteed (Bruhn, 2002). It does not help that in trying to breed for higher soluble solids; yield is usually compromised and may fall below the profitability threshold for a tomato crop (Stevens, 1986). As a result the cost benefit ratio currently tilts in favor of non-taste related traits. Therefore, selection of cultivars with elevated soluble solid and positive correlation with yield would be advantageous. Considering the fact, the present study was undertaken to determine the genetic association between different morphological and nutritional traits for further development and selection of superior nutritional rich high yielding tomato genotypes.

\section{Materials and Methods}

The experiment was conducted at the experimental field, Department of Genetics and Plant Breeding, Bangladesh Agricultural University (BAU), Mymensingh during winter season (October 2017 to March 2018) on an upland soil. The experimental site was situated in the sub tropical climate zone, characterized by heavy rainfall during the months from May to September and scanty rainfall in the rest of the year. The experimental material consisted of 21 advanced tomato genotypes (Tm-131, Tm-134, Tm-181, Tm-206, Tm-219, Tm-299, Tm-337, V100589, V1005582, V1006484, V1006282, V1006015, V1057583, V100686, V1007282, V1005584, C-11, C-71, WP-7, Homeastid) and 4 varieties (BINA tomato 4, BINA tomato 8, BINA tomato 9 and BINA tomato 10) were planted in healthy plot to assess different yield and nutritional quality attributing traits. The experimental seed material was sown in pots on 10 October, 2017 and 30 days old seedlings were transplanted to the main field on 10 November, 2017 with spacing of $60 \mathrm{~cm} \mathrm{x} 40 \mathrm{~cm}$. Before transplanting the plot was brought to fine tilth by ploughing and harrowing. The recommended doses of fertilizers such as cowdung $(10,000 \mathrm{~kg} / \mathrm{ha})$, Urea $(250$ $\mathrm{kg} / \mathrm{ha})$, TSP (250 kg/ha) and MP (150 kg/ha) were applied during cultivation. The experiment was conducted using Randomized Complete Block Design (RCBD) with three replications. Correlation, path analysis and principal component analysis were computed with the help of computer software MSTATC
(Genetic analysis), BASICA (path coefficient analysis) and Minitab 17 (PCA analysis) following the techniques suggested by Johnson et al. (1955); Hanson et al. (1956); Miller et al., (1991) and Lynch and Walsh (1998).

\section{Results and Discussion}

Analysis of variance for different yield and quality contributing components presented in the Table 1 revealed significant differences among genotypes for all the characters. Shravan et al., (2004) found significant varietal differences for fruit weight by using thirty tomato varieties. Singh \& Raj (2004) and Barman et al., (1995) had also reported the variability of tomato genotypes for fruit/bunch, individual fruit diameter and individual fruit weight, and their direct effects on final yield. Genotypic and phenotypic coefficient of variation and heritability revealed high range for most of traits studied (Table 1). High heritability for plant height on $1^{\text {st }}$ leaf appearance, soluble solid in green and red tomato, total phenolic content, fruit diameter and weight, and seed/fruit indicated less influence of environments that could be exploited through simple selection from this material to improve yield as suggested by Mohanty, (2003). Low to medium heritability for plant height, leaf chlorophyll content, and $\mathrm{p}^{\mathrm{H}}$ in red tomato suggested a careful selection from the material for enhancing the genetic portion of variation that can also be attained through addition of superior germplasm (Johnson et al., 1955).

The genotypic and phenotypic correlations among all the characters are presented in Table 2 . In most of the cases genotypic and phenotypic correlation coefficients were of the same directions but the former were slightly higher in magnitude indicating low influence of environments that enhanced the acceptance of these findings (Shravan et al., 2004; Nakawuka \& Adipala, 1999). The results revealed that the days to first fruit maturity showed positive and significant correlation (at $\mathrm{p}=0.01)$ with yield/plant $(0.315)$, followed by fruit weight (0.303). It also had positive and significant (at $\mathrm{p}$ $=0.05)$ correlation with fruit diameter $(0.278)$ and days to first branching (0.274) whereas, fruit/bunch showed positive and significant correlation (at $\mathrm{p}=0.01$ ) with yield/plant (0.329). It is expected that the more the bunch number in a plant, such plant will produce more fruits resulting in more fruit weight (Singh \& Raj, 2004). Plant height showed positive and significant correlation (at $\mathrm{p}=0.001$ ) with individual fruit diameter (0.372). It also showed positive and significant correlation (at $\mathrm{p}=0.01)$ with $\mathrm{p}^{\mathrm{H}}$ in green tomato juice $(0.374)$ followed by yield/plant $(0.353)$, chlorophyll content $(0.352)$, and individual fruit weight (0.349). Plant height also showed positive and significant correlation (at $\mathrm{p}=0.05$ ) with soluble solid in red tomato $(0.436)$ followed by soluble solid in green tomato (0.254). Individual fruit diameter showed positive and significant correlation (at $\mathrm{p}=0.001$ ) with individual fruit weight (0.839) followed by yield/plant (0.660). It also showed negative and 
significant correlation (at $\mathrm{p}=0.05)$ with soluble solids in green tomato $(-0.285)$ whereas, individual fruit weight showed positive and significant correlation (at $\mathrm{p}=0.001$ ) with yield/plant (0.766) followed by seed/fruit (0.572). It also showed negative and significant correlation (at $\mathrm{p}=$ $0.01)$ with total phenolic content $(-0.297)$ and (at $\mathrm{p}=0.05)$ with soluble solids in green tomato $(-0.248)$ and soluble solids in red tomato $(-0.129)$. This negative relationship with total soluble solids suggests that there may be competition for resources between total soluble solids and other components (Maršić et al., 2011). Leaf chlorophyll content showed positive and significant correlation (at $\mathrm{p}=0.01$ ) with soluble solid in green tomato (0.350) whereas, total phenolic content showed positive and significant correlation (at $\mathrm{p}=0.05$ ) with soluble solid in green tomato (0.293). These results are in conformity with the findings of Abedin and Khan (1986); Reddy and Reddy (1992); McGillivary and Clemente (1956); Stevens and Rudish (1978); Maršić et al., (2011); Ara et al., (2009) and Joshi et al., (1998). The correlation results obtained in the present study indicated that parameters viz., plant height, seed/fruit, fruits/bunch, average fruit weight, plant height are the important components of yield. Therefore, to increase the yield in tomato, selection for above mentioned parameters can be carried out. On the other hand, to increase nutrition value as well as higher yield in tomato selection, we need to increase the soluble solids in tomato which was positively correlated with chlorophyll content and total phenolic content that has free radical scavenging capacity. Though the soluble solids in tomato was inversely correlated with yield, which can be overcome through selective breeding method, genetic engineering or mutation to broaden the genetic base for selection to improve nutrition value with fruit yield (Arshad et al., 2005).

Table 1. Genetic parameters for various morphological and biochemical characteristics in 25 tomato genotypes

\begin{tabular}{|c|c|c|c|c|c|c|c|c|}
\hline Characters & MS & $\begin{array}{c}\text { Genotypic } \\
\text { variance }\left(\sigma^{2} \mathrm{~g}\right)\end{array}$ & $\begin{array}{c}\text { Phenotypic } \\
\text { variance }\left(\sigma^{2} p\right)\end{array}$ & $\begin{array}{l}\text { Heritability } \\
(\%)\end{array}$ & GA & $\begin{array}{l}\text { GA } \\
(\%)\end{array}$ & $\begin{array}{c}\mathrm{GCV} \\
(\%)\end{array}$ & $\begin{array}{r}\mathrm{PCV} \\
(\%)\end{array}$ \\
\hline $\begin{array}{l}\text { Plant height on } 1^{\text {st }} \text { leaf } \\
\text { appearance }(\mathrm{cm})\end{array}$ & $1.36^{* * *}$ & 0.43 & 0.49 & 89.2 & 1.28 & 24.58 & 12.64 & 13.38 \\
\hline Days to first branching & $38.08 * * *$ & 8.13 & 21.82 & 37.25 & 3.58 & 6.81 & 5.42 & 8.88 \\
\hline Days to first flowering & $13.74 *$ & 1.95 & 9.82 & 19.92 & 1.28 & 2.04 & 1.59 & 4.99 \\
\hline Days to first fruiting & $34.95 * * *$ & 7.52 & 19.91 & 37.76 & 3.46 & 4.42 & 3.50 & 5.70 \\
\hline Days to first fruit maturity & $35.53 * * *$ & 9.36 & 16.80 & 55.71 & 4.70 & 4.08 & 2.65 & 3.55 \\
\hline Fruit/bunch & $2.24 *$ & 0.32 & 1.60 & 20.12 & 0.52 & 10.41 & 11.30 & 25.19 \\
\hline Individual fruit diameter & $16.22 * * *$ & 5.25 & 5.72 & 91.85 & 4.52 & 32.60 & 16.51 & 17.23 \\
\hline Individual fruit weight (gm) & $703.55 * * *$ & 234.02 & 235.49 & 99.37 & 31.41 & 72.15 & 35.14 & 35.25 \\
\hline Plant height $(\mathrm{cm})$ & $2305.8 * * *$ & 688.06 & 929.66 & 74.01 & 46.47 & 48.13 & 27.17 & 31.58 \\
\hline Seed/fruit & $1251.46^{* * *}$ & 411.67 & 428.12 & 96.15 & 40.98 & 53.18 & 26.33 & 26.85 \\
\hline $\mathrm{p}^{\mathrm{H}}$ in green tomato juice & $0.38 * * *$ & 0.09 & 0.19 & 51.57 & 0.46 & 9.93 & 6.71 & 9.35 \\
\hline $\mathrm{p}^{\mathrm{H}}$ in red tomato juice & $0.32 * * *$ & 0.09 & 0.13 & 74.05 & 0.55 & 12.36 & 6.98 & 8.11 \\
\hline $\begin{array}{l}\text { Leaf chlorophyll content (SPAD } \\
\text { unit) }\end{array}$ & $27.87 * * *$ & 8.56 & 10.74 & 79.69 & 5.38 & 16.68 & 9.07 & 10.16 \\
\hline $\begin{array}{l}\text { Total phenolic content }(\mu \mathrm{g} / \mathrm{g} \text { dry } \\
\text { weight) }\end{array}$ & $623778 * * *$ & 207872 & 208034 & 99.92 & 938.8 & 48.84 & 23.72 & 23.73 \\
\hline $\begin{array}{l}\text { Soluble solids in green tomato }(\% \\
\text { Brix) }\end{array}$ & $1.20 * * *$ & 0.39 & 0.42 & 93.23 & 1.24 & 28.64 & 14.40 & 14.91 \\
\hline $\begin{array}{l}\text { Soluble solid in red tomato ( } \% \\
\text { Brix) }\end{array}$ & $4.57 * * *$ & 1.51 & 1.54 & 98.58 & 2.52 & 52.17 & 25.51 & 25.69 \\
\hline Yield/plant (kg) & $1.17 * * *$ & 0.35 & 0.46 & 75.21 & 1.05 & 61.40 & 34.69 & 40 \\
\hline
\end{tabular}

* indicates $5 \%$ level of significance; *** indicates $0.1 \%$ level of significance

MS = mean sum of square, $\mathbf{P C V}=$ Phenotypic Coefficient of Variation, $\mathbf{G C V}=$ Genotypic Coefficient of Variation, $\mathbf{G A}=$ Genetic Advance, $\mathbf{G A}$ $(\%)=$ Genetic advance in percentage of mean 
Table 2. Genotypic $\left(r_{g}\right)$ and phenotypic $\left(r_{p}\right)$ correlation coefficient among different yield and soluble solids contributing characters in 25 tomato genotypes

\begin{tabular}{|c|c|c|c|c|c|c|c|c|c|c|c|c|c|c|c|c|c|}
\hline Charc. & Cor & DFFl & DFFr & DFM & PHLA & $\mathrm{Fr} / \mathrm{B}$ & $\mathrm{PH}$ & IFD & IFW & $\mathrm{Sd} / \mathrm{Fr}$ & $\mathrm{pH} / \mathrm{G}$ & $\mathrm{pH} / \mathrm{R}$ & Chl.C & TPC & SS/G & SS/R & $\mathrm{Y} / \mathrm{P}$ \\
\hline \multirow{2}{*}{$\overline{\mathrm{DFB}}$} & $r_{g}$ & $1.049 * * *$ & $0.451 *$ & $0.209 *$ & -0.263 & $-0.548 *$ & -14.019 & 0.453 & $0.465^{*}$ & 0.461 & 0.167 & 0.188 & 0.081 & -0.267 & $-0.538 *$ & -0.056 & 0.268 \\
\hline & $\mathrm{r}_{\mathrm{p}}$ & $0.454 * * *$ & $0.289^{*}$ & $0.274 *$ & -0.195 & $-0.252 *$ & -0.177 & 0.218 & $0.291 *$ & 0.214 & 0.010 & 0.072 & 0.007 & -0.162 & $-0.293^{*}$ & -0.037 & 0.059 \\
\hline \multirow{2}{*}{ DFFl } & $r_{g}$ & & $1.149 * * *$ & -0.010 & $-0.518^{*}$ & -0.940 & 0.084 & -0.138 & 0.217 & 0.220 & $-0.007^{*}$ & 0.050 & 0.177 & -0.168 & -0.093 & 0.249 & -0.013 \\
\hline & $r_{p}$ & & $0.445^{* *}$ & 0.146 & $-0.244 *$ & -0.082 & 0.050 & -0.061 & 0.095 & 0.110 & $-0.037^{*}$ & 0.013 & 0.166 & -0.078 & -0.038 & 0.111 & -0.032 \\
\hline \multirow[t]{2}{*}{ DFFr } & $r_{g}$ & & & 0.001 & $-0.479^{*}$ & -0.582 & 0.349 & -0.379 & -0.242 & -0.110 & 0.080 & -0.214 & 0.103 & 0.077 & 0.120 & $0.412 *$ & -0.244 \\
\hline & $r_{p}$ & & & 0.228 & $-0.293^{*}$ & -0.162 & 0.157 & -0.162 & -0.142 & -0.029 & 0.024 & -0.054 & 0.196 & 0.044 & 0.094 & $0.245^{*}$ & -0.111 \\
\hline \multirow[t]{2}{*}{ DFM } & $r_{g}$ & & & & 0.108 & 0.282 & -0.142 & $0.351 *$ & $0.417 * *$ & 0.034 & -0.025 & 0.089 & -0.137 & -0.195 & -0.027 & 0.073 & $0.450 * *$ \\
\hline & $r_{p}$ & & & & 0.069 & 0.015 & -0.142 & $0.278^{*}$ & $0.303^{* *}$ & 0.050 & -0.100 & 0.087 & -0.041 & -0.146 & 0.012 & 0.040 & $0.315^{* *}$ \\
\hline \multirow[t]{2}{*}{ PHLA } & $r_{g}$ & & & & & 0.210 & $0.320^{* *}$ & 0.117 & 0.071 & -0.013 & 0.338 & $0.420^{* *} *$ & 0.114 & 0.046 & -0.140 & -0.048 & 0.091 \\
\hline & $r_{p}$ & & & & & 0.082 & $0.303 * *$ & 0.109 & 0.067 & -0.013 & 0.229 & $0.331 * *$ & 0.082 & 0.044 & -0.125 & -0.046 & 0.116 \\
\hline \multirow[t]{2}{*}{$\mathrm{Fr} / \mathrm{B}$} & $r_{g}$ & & & & & & 0.042 & 0.249 & 0.307 & 0.247 & 0.056 & -0.187 & 0.113 & -0.275 & 0.448 & -0.214 & $0.312^{* *}$ \\
\hline & $r_{p}$ & & & & & & 0.023 & 0.099 & 0.119 & 0.126 & 0.035 & -0.061 & 0.131 & -0.125 & 0.170 & -0.093 & $0.329 * *$ \\
\hline \multirow[t]{2}{*}{ PH } & $\mathrm{r}_{\mathrm{g}}$ & & & & & & & $0.452 * * *$ & $0.418^{* *}$ & -0.151 & $0.574 * *$ & 0.204 & $0.492 * *$ & 0.230 & $0.326^{*}$ & $0.510^{*}$ & $0.448^{* *}$ \\
\hline & $r_{p}$ & & & & & & & $0.372 * * *$ & $0.349 * *$ & -0.143 & $0.374 * *$ & 0.097 & $0.352 * *$ & 0.198 & $0.254 *$ & $0.436^{*}$ & $0.353^{* *}$ \\
\hline \multirow[t]{2}{*}{ IFD } & $\mathrm{r}_{\mathrm{g}}$ & & & & & & & & $0.875^{* * *} *$ & $0.342^{* *}$ & -0.042 & -0.031 & -0.006 & -0.216 & $-0.303^{*}$ & 0.001 & $0.785 * * *$ \\
\hline & $r_{p}$ & & & & & & & & $0.839 * * *$ & $0.327^{* *} *$ & -0.047 & -0.021 & 0.041 & -0.206 & $-0.285^{*}$ & -0.004 & $0.660 * * *$ \\
\hline \multirow[t]{2}{*}{ IFW } & $r_{g}$ & & & & & & & & & $0.587 * * *$ & -0.213 & 0.028 & 0.013 & $-0.298 * *$ & $-0.261 *$ & $-0.130 *$ & $0.897 * * *$ \\
\hline & $r_{p}$ & & & & & & & & & $0.572 * * *$ & -0.139 & 0.028 & 0.046 & $-0.297 * *$ & $-0.248 *$ & $-0.129 *$ & $0.766^{* * *}$ \\
\hline \multirow[t]{2}{*}{$\mathrm{Sd} / \mathrm{Fr}$} & $r_{g}$ & & & & & & & & & & $-0.387 * *$ & 0.043 & 0.001 & $-0.366^{*}$ & $-0.266^{*}$ & $0.297 *$ & $0.600 * * *$ \\
\hline & $r_{p}$ & & & & & & & & & & $-0.276^{* *}$ & 0.035 & 0.013 & $-0.365^{*}$ & $-0.250 *$ & $0.284 *$ & $0.519 * * *$ \\
\hline \multirow{2}{*}{$\mathrm{p}^{\mathrm{H} / \mathrm{G}}$} & $\mathrm{r}_{\mathrm{g}}$ & & & & & & & & & & & $0.519 * * *$ & 0.362 & 0.026 & 0.282 & 0.221 & -0.278 \\
\hline & $r_{p}$ & & & & & & & & & & & $0.296 * * *$ & 0.196 & 0.017 & 0.180 & 0.158 & -0.135 \\
\hline \multirow{2}{*}{$\mathrm{p}^{\mathrm{H} / \mathrm{R}}$} & $r_{g}$ & & & & & & & & & & & & 0.073 & 0.260 & 0.022 & -0.034 & -0.103 \\
\hline & $r_{p}$ & & & & & & & & & & & & 0.075 & 0.215 & 0.026 & -0.025 & -0.090 \\
\hline \multirow[t]{2}{*}{ Chl.C } & $r_{g}$ & & & & & & & & & & & & & 0.087 & $0.408 * *$ & 0.090 & 0.011 \\
\hline & $\mathrm{r}_{\mathrm{p}}$ & & & & & & & & & & & & & 0.077 & $0.350 * *$ & 0.067 & -0.001 \\
\hline \multirow[t]{2}{*}{ TPC } & $\mathrm{r}_{\mathrm{g}}$ & & & & & & & & & & & & & & $0.304 *$ & 0.024 & -0.121 \\
\hline & $r_{p}$ & & & & & & & & & & & & & & $0.293 *$ & 0.023 & -0.107 \\
\hline \multirow[t]{2}{*}{$\mathrm{SS} / \mathrm{G}$} & $r_{g}$ & & & & & & & & & & & & & & & 0.203 & -0.187 \\
\hline & $\mathrm{r}_{\mathrm{p}}$ & & & & & & & & & & & & & & & 0.193 & -0.157 \\
\hline \multirow[t]{2}{*}{$\mathrm{SS} / \mathrm{R}$} & $r_{g}$ & & & & & & & & & & & & & & & & -0.124 \\
\hline & $r_{p}$ & & & & & & & & & & & & & & & & -0.106 \\
\hline
\end{tabular}

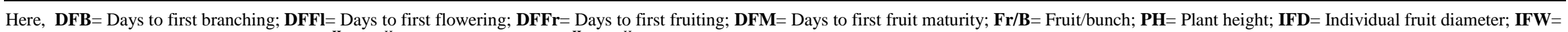
Individual fruit weight, $\mathbf{S d} / \mathbf{F r}=$ Seed/fruit; $\mathbf{p}^{\mathrm{H}} / \mathbf{G}=\mathrm{p}^{\mathrm{H}}$ in green tomato juice; $\mathbf{p}^{\mathrm{H}} / \mathbf{R}=\mathrm{p}^{\mathrm{H}}$ in red tomato juice; $\mathbf{P H L A}=$ Plant height on first leaf appearance; $\mathbf{C h l} . \mathbf{C}=\mathrm{Leaf}$ chlorophyll content, $\mathbf{T P C}=$ Total phenolic content $; \mathbf{S S} / \mathbf{G}=$ Soluble solids in green tomato, $\mathbf{S S} / \mathbf{R}=$ Soluble solids in red tomato; $\mathbf{Y} / \mathbf{P}=$ Yield/plant 
The estimates of direct and indirect effects of different characters on the fruit yield/plant and soluble solid (sugar) are presented character wise (Table 3 and 4). The results revealed that the path analysis of yield/plant and soluble solids (sugar) with its component traits presented diagonally depicted direct effects of the characters towards their correlation with yield/plant and soluble solid, while all other off diagonal estimates showed indirect effects of the characters towards their correlation with yield/plant and soluble solid. Individual fruit weight (0.539) followed by plant height (0.313), total phenolic content (0.304), seed/fruit (0.279), fruit/bunch (0.226), days to first fruit maturity (0.186), $\mathrm{p}^{\mathrm{H}}$ in green tomato juice $(0.170)$ and plant height on first leaf appearance $(0.145)$ were showing positive direct effects on yield/plant while other parameters like $\mathrm{p}^{\mathrm{H}}$ in red tomato juice $(-0.228)$ followed by days to first branching $(-0.155)$, soluble solid in red tomato $(-0.094)$, soluble solid in green tomato (-0.088), days to first flowering (-0.0107) and individual fruit diameter $(-0.0004)$ were showing direct negative effect (Table 4). On the other hand, days to first flowering (0.347) followed by leaf chlorophyll content $(0.216)$ and $\mathrm{p}^{\mathrm{H}}$ in red tomato juice $(0.101)$ were showing positive direct effects on soluble solid in red tomato while other parameters like individual fruit weight $(-1.015)$ followed by individual fruit diameter (-0.847), plant height ($0.511)$, fruit/bunch $(-0.236)$, days to first branching ($0.233)$, days to first fruit maturity $(-0.075)$ and total phenolic content $(-0.054))$ were showing direct negative effect (Table 4). The results are in accordance with the reports of Mohanty (2002, 2003); Singh (2005); Rani et al., (2008); Ara et al., (2009); Mayavel et al., (2005); Tanksley (2004).

Table 3. Partitioning phenotypic correlation into direct (Bold) and indirect effects of 16 characters on yield/plant in 25 tomato genotypes

\begin{tabular}{|c|c|c|c|c|c|c|c|c|c|c|c|c|c|c|c|c|c|}
\hline Char. & DFB & DFFl & DFFr & DFM & HLA & Fr/B & PH & IFD & IFW & $\mathrm{Sd} / \mathbf{F r}$ & $\mathbf{p}^{\mathrm{H}} / \mathrm{G}$ & $\mathbf{p}^{\mathrm{H}} / \mathbf{R}$ & Chl.C & TPC & SS/G & SS/R & $\mathbf{Y} / \mathbf{P}$ \\
\hline$\overline{\mathrm{DFB}}$ & -0.155 & -0.004 & 0.015 & 0.050 & .027 & -0.056 & 0.053 & .00009 & 0.156 & 0.058 & 0.001 & -0.015 & 0.0002 & 0.048 & 0.025 & 0.002 & 059 \\
\hline DFFl & -0.070 & $-\mathbf{0 . 0 1 0 7}$ & 0.024 & 026 & 034 & .018 & .015 & 00002 & 048 & 0.030 & .005 & -0.002 & 04 & .021 & 0.002 & 10 & 032 \\
\hline DFFr & -0.043 & -0.004 & 0.056 & 0.041 & & -0.036 & & 0007 & 75 & & & & & 2 & 97 & 2 & 1 \\
\hline DFM & -0.042 & -0.001 & 0.012 & 0.186 & 0.008 & 0.002 & 0.043 & 0.0001 & 0.161 & 0.013 & -0.017 & -0.018 & -0.001 & -0.042 & -0.0008 & 0.003 & $315^{* * *}$ \\
\hline HLA & 0.029 & 0. & 16 & 1 & & 8 & 94 & 0004 & 32 & & & & & & & & \\
\hline $\mathrm{Fr} / \mathrm{B}$ & 0.038 & 0.0008 & -0.009 & 0.001 & 0.011 & 0.226 & -0.006 & -0.00004 & 0.059 & 0.033 & 0.005 & 0.013 & 0.003 & -0.036 & -0.015 & -0.085 & $.329 * *$ \\
\hline $\mathrm{PH}$ & 0.026 & -0 . & 0.008 & -0.026 & 43 & 04 & 13 & 001 & & & & & & 0.057 & -0.022 & 0.040 & $53 * *$ \\
\hline IFD & -0.032 & 0.0006 & -0.009 & 0.050 & 0.014 & 0.020 & 0.116 & -0.0004 & 0.448 & 0.089 & -0.006 & 0.004 & 0.001 & -0.060 & 0.024 & -0.0003 & 0.660 *** \\
\hline IFW & -0.045 & -0.0009 & -0.007 & 0.056 & 0.008 & 0.024 & 0.106 & -0.0003 & 0.539 & 0.159 & -0.022 & -0.004 & 0.001 & -0.088 & 0.021 & -0.011 & $0.766^{* * * *}$ \\
\hline $\mathrm{Sd} / \mathrm{Fr}$ & & & & & & 0.027 & & & & & & & & -0.109 & & & $0.519^{* * *} *$ \\
\hline $\mathrm{p}^{\mathrm{H}} / \mathrm{G}$ & -0.001 & 0.0003 & 0.001 & -0.018 & 032 & 0.006 & -0.116 & 0.00001 & -0.070 & -0.075 & 70 & -0.066 & 0.005 & 0.003 & -0.015 & 0.014 & -0.135 \\
\hline $\mathrm{p}^{\mathrm{H}} / \mathrm{R}$ & -0.010 & -0.0001 & -0.002 & 0.014 & & -0.013 & -0.028 & 0.000009 & 0.010 & 0.008 & & & 02 & 0.063 & -0.001 & -0.001 & 90 \\
\hline Chl.C & -0.001 & -0.001 & 0.010 & -0.007 & 011 & 0.029 & -0.109 & -0.00001 & 0.021 & 0.002 & 32 & -0.015 & 0.0304 & 0.021 & -0.031 & 0.005 & -0.001 \\
\hline TPC & 0.024 & 0.0007 & 0.002 & -0.026 & .005 & -0.027 & -0.059 & 0.00009 & -0.156 & -0.100 & & -0.047 & 0.002 & 0.304 & -0.025 & 0.001 & 107 \\
\hline $\mathrm{SS} / \mathrm{G}$ & 0.045 & 0.0003 & 0.005 & 0.001 & -0.017 & 0.038 & -0.078 & 0.0001 & -0.129 & -0.069 & 30 & -0.004 & 0.010 & 0.088 & -0.088 & 0.017 & -0.157 \\
\hline SS/R & 0.004 & -0.001 & 0.013 & 0.007 & -0.005 & -0.020 & -0.134 & 0.000001 & -0.064 & 0.055 & 0.025 & 0.004 & 0.001 & 0.006 & -0.016 & -0.094 & -0.106 \\
\hline
\end{tabular}

Residual effect $=0.189$, *indicates $5 \%$ level of significance; $* *$ indicates $1 \%$ level of significance; $* * *$ indicates $0.1 \%$ level of significance

Here, $\mathbf{D F B}=$ Days to first branching; $\mathbf{D F F l}=$ Days to first flowering; $\mathbf{D F F r}=$ Days to first fruiting; $\mathbf{D F M}=\mathbf{D a y s}$ to first fruit maturity; $\mathbf{F r} / \mathbf{B}=$ Fruit/bunch; $\mathbf{P H}=$ Plant height; IFD= Individual fruit diameter; $\mathbf{I F W}=$ Individual fruit weight, $\mathbf{S d} / \mathbf{F r}=\mathrm{Seed} / \mathrm{fruit} ; \mathbf{p}^{\mathbf{H}} / \mathbf{G}=\mathrm{p}^{\mathrm{H}}$ in green tomato juice; $\mathbf{p}^{\mathbf{H}} / \mathbf{R}=\mathrm{p}^{\mathrm{H}}$ in red tomato juice; $\mathbf{P H L A}=$ Plant height on first leaf appearance; $\mathbf{C h l} . \mathbf{C}=$ Leaf chlorophyll content, $\mathbf{T P C}=\mathrm{Total}$ phenolic content ; $\mathbf{S S} / \mathbf{G}=$ Soluble solids in green tomato, $\mathbf{S S} / \mathbf{R}=$ Soluble solids in red tomato; $\mathbf{Y} / \mathbf{P}=$ Yield/plant

Table 4. Partitioning of phenotypic correlation into direct (Bold) and indirect effects of 10 characters on soluble solid (sugar) in 25 tomato genotypes

\begin{tabular}{|c|c|c|c|c|c|c|c|c|c|c|c|}
\hline Characters & DFB & DFFl & DFM & Fr/B & PH & IFD & IFW & $\mathbf{p}^{\mathbf{H}} / \mathbf{R}$ & ChlC & TPC & SS/R \\
\hline DFB & -0.233 & 0.156 & -0.020 & 0.059 & 0.087 & 0.177 & -0.294 & 0.007 & 0.001 & 0.008 & -0.037 \\
\hline DFFI & -0.105 & 0.347 & -0.010 & -0.018 & -0.025 & -0.050 & -0.091 & 0.001 & 0.034 & 0.003 & 0.111 \\
\hline DFM & -0.063 & 0.048 & -0.075 & -0.002 & 0.071 & 0.228 & -0.304 & 0.008 & -0.008 & 0.007 & 0.040 \\
\hline $\mathrm{Fr} / \mathrm{B}$ & 0.058 & -0.027 & -0.0007 & -0.236 & -0.010 & 0.076 & -0.111 & -0.006 & 0.028 & 0.006 & -0.093 \\
\hline PH & 0.039 & 0.017 & 0.010 & -0.004 & -0.511 & 0.313 & 0.345 & 0.009 & 0.075 & -0.010 & $0.436 *$ \\
\hline IFD & -0.049 & -0.020 & -0.020 & -0.021 & -0.189 & -0.847 & -0.845 & -0.002 & 0.008 & 0.010 & -0.004 \\
\hline IFW & -0.067 & 0.031 & -0.022 & -0.025 & 0.174 & 0.703 & -1.015 & 0.002 & 0.008 & 0.015 & $-0.129 *$ \\
\hline $\mathbf{p}^{\mathbf{H}} / \mathbf{R}$ & -0.016 & 0.003 & -0.006 & 0.014 & -0.046 & -0.016 & -0.020 & 0.101 & 0.015 & -0.011 & -0.025 \\
\hline ChlC & -0.001 & 0.055 & 0.003 & -0.030 & -0.179 & 0.033 & -0.040 & 0.007 & 0.216 & -0.003 & 0.067 \\
\hline TPC & 0.037 & -0.024 & 0.010 & 0.028 & -0.097 & -0.169 & 0.294 & 0.021 & 0.015 & -0.054 & 0.023 \\
\hline
\end{tabular}

Residual effect $=0.307, *$ indicates $5 \%$ level of significance

In the present investigation, after analyzing principal component (PC), five main principal components showed more than 1 Eigen value and exhibited about $75.1 \%$ cumulative variability (Table 5). The first components accounted for $25.1 \%$ of total variability and the most important traits were: individual fruit weight $(0.450)$, yield/plant $(0.404)$ with positive coefficient and total phenolic contents $(0.224)$ with negative coefficient indicating inverse relationship between variables. The first component representing the significance of this PC 
for yield related traits. Days to first flowering (0.497) and days to first fruiting (0.467) both with positive coefficient were the important traits of PC2 which contributes $16.3 \%$ of total variability indicating usefulness of this PC for changing duration of the plant maturity. The third principal component attributed $13.6 \%$ and showed no contrast among the studied traits with almost all negative coefficients. The fourth main component explained $10.9 \%$ of total variability with fruit/bunch (0.458) and $\mathrm{pH}(0.439)$ as the most important traits. The PC5 which covered about $9.2 \%$ of total variability and soluble solid contents in fruits $(0.326 \&$ $0.440)$ and days to fruit maturity (0.348) were the important contributing traits, indicating usefulness of this PC for nutritional rich genotypes selection. Henareh et al. (2015) conducted an experiment on 97 tomato land races where they found three main components which explained $71.6 \%$ of total variability in principal component analysis. In another study, Chernet et al. (2014) tested 36 tomato genotypes where they obtained six principal components explaining $83.03 \%$ of total variability. Soluble solid content and $\mathrm{pH}$ are of important components for the taste of tomato fruits (Rodica et al., 2008) needs to be considered for quality tomato fruits.

Table 5. Principal components and their coefficients of 17 important tomato traits

\begin{tabular}{lccccc}
\hline Variable & \multicolumn{5}{c}{ Main components } \\
\cline { 2 - 6 } & PC1 & PC2 & PC3 & PC4 & PC5 \\
\hline DFB & 0.165 & 0.349 & -0.139 & 0.296 & 0.185 \\
DFF1 & 0.028 & 0.497 & -0.169 & -0.096 & -0.040 \\
DFFr & -0.109 & 0.467 & -0.237 & -0.056 & 0.055 \\
DFM & 0.184 & 0.150 & -0.205 & 0.065 & 0.348 \\
PHLA & 0.005 & -0.346 & -0.281 & 0.272 & -0.238 \\
Fr/B & 0.093 & -0.244 & -0.133 & -0.458 & -0.112 \\
PH & -0.291 & -0.015 & -0.382 & -0.059 & -0.361 \\
SS/G & -0.213 & -0.067 & -0.189 & -0.262 & 0.326 \\
SS/R & -0.266 & 0.237 & -0.158 & 0.099 & 0.440 \\
IFD & 0.414 & -0.085 & -0.132 & 0.044 & 0.124 \\
IFW & 0.450 & -0.011 & -0.188 & 0.005 & -0.027 \\
Sd/Fr & 0.312 & 0.094 & -0.116 & -0.075 & -0.405 \\
p $/ G$ & -0.161 & -0.222 & -0.364 & 0.242 & 0.111 \\
p/R & -0.053 & -0.185 & -0.326 & 0.439 & 0.179 \\
Y/P & 0.404 & -0.101 & -0.176 & -0.157 & -0.101 \\
Chl.C & -0.060 & -0.016 & -0.263 & -0.320 & 0.161 \\
TPC & -0.224 & -0.077 & -0.013 & 0.012 & 0.282 \\
Eigen values & 3.9 & 2.37 & 1.88 & 1.51 & 1.22 \\
\% Total & 25.1 & 16.3 & 13.6 & 10.9 & 9.2 \\
Variance & & & & & \\
Cumulative & 25.1 & 41.4 & 55.0 & 65.9 & 75.1 \\
(\%) & & & & & \\
\hline
\end{tabular}

\section{Conclusion}

The characters showing high direct effect on yield/plant and soluble solid indicated that direct selection for these traits might be effective and there is a possibility of improving yield/plant and soluble solid in tomato through selection based on these characters. By analyzing seventeen different morphological and organoleptic characters, the presence of wide diversity among the genotypes was found. It was observed from the study of heritability, genetic advance, correlation coefficient, path coefficient analyses and principal component analysis that the individual fruit weight and diameter was the most important character related to the yield/plant than other traits; whereas, chlorophyll content and total phenolic content was the most important trait for soluble solids, although there is a bit inverse relations between soluble solids and yield/plant. The genotypes exhibited moderate to higher fruit yield with soluble solid (sugar) and other desirable traits; hence these are suggested to test under potential areas for identification of best cultivar for general cultivation. This analysis could be beneficial for the further breeding program for utilizing the genotypes and for effective selection for boosting yield and flavor in tomato.

\section{Acknowledgements}

This work was supported by University Grants Commission of Bangladesh (UGC) to GHMS (2017/4773/UGC)

\section{References}

Abedin, J. and Khan, S.H. 1986. Study of the morphogenetic divergence in tomato. Bangl. J. Agric. Res. 11:(1), 39-47.

Arshad, M., Ghafoor, A. and Qureshi, A.S. 2005. Inheritance of qualitative traits and their linkage in blackgram (Vigna mungo (L.) Hepper). Pak. J. Bot. 37: (1), 41-46.

Ara, A.R., Narayan, N. and Khan, S.H. 2009. Genetic variability and selection parameters for yield and quality attributes in tomato. Ind. J. Hort .66:73-78.

Alvarenga, M.A.R. 2004. Tomate: Produção em Campo, em Casa-deVegetação e em Hidroponia; UFLA: Lavras, MG, Brazil. 400 .

Bhutani, R.D. and Kallo, G. 1983. Genetics of carotenoids and lycopene in tomato (L. esculentum. Mill). Genet. Agrar. 37: $1-6$.

Baldwin, E.A., Scott, J.W., Einstein, M.A., Malundo, T.M.M., Carr, B.T., Shewfelt, R.L. and Tandon, K.S. 1998. Relationship between sensory and instumental analysis for tomato flavor. J. Amer. Soc. Hort. Sci. 125: 906-915.

Brauss, M.S., Linforth, R.S. and Taylor, A.J. 1998. Effect of variety, time of eating and fruit-to-fruit variation on volatile release during eating of tomato fruits (Lycopersicon esculentum). J. Agric. Fd. Chem. 46: 2287-2292.

Barman, D., Sharma, C.K., Singh, I.P., Sardana, S.D. 1995. Genetic variability in exotic lines of tomato (Lycopersicon esculentum Mill.) in off season. Int. J. Trop. Agric. 13: 265-268.

BBS (Bangladesh Bureau of Statistics). 2008. Statistical Yearbook of Bangladesh. Stat. Div. Min. Planning, Dhaka, Bangladesh. 147.

BBS (Bangladesh Bureau of Statistics). 2016. Statistical Yearbook of Bangladesh. Stat. Div. Min. Planning, Dhaka, Bangladesh. 145.

Bruhn, C. M. 2002. Consumer issues in quality and safety. Third ed. University of California Agricultural and Natural Resources, Oakland.

Causse, M., Saliba-Colombani, V., Lesschaeve, I. and Buret, M. 2001. Genetic analysis of organoleptic quality in fresh market tomato. Theor. Appl. Genet. 102: 273-283.

Chernet, S., Belew, D. and Abay, F. 2014. Genetic diversity studies for quantitative traits of tomato (Solanum lycopersicon L.) genotypes in Western Tigray, Northern Ethiopia. J. Pl. Breed. Crop Sci. 6: (9), 105-113.

Hanson, G., Robinson, H.F. and Comstock, R.E. 1956. Biometrical studies on yield in segregating population of Korean Lespedeza. J. Agron. 48: 268-274.

Henareh, M., Dursun, A. and Mandoulakani, B.A. 2015. Genetic diversity in tomato landraces collected from Turkey and 
Iran revealed by morphological characters. Acta Sci. Polonorum Hort. Cult. 14: (2), 87-96.

Islam, M.S. and Khan, S. 1991. Variability and character association in tomato. Bangl. J. Pl. Breed. \& Genet. 4: (1\&2), 49-53.

Johnson, H.W., Robinson, F.P.F. and Comstock, R.E. 1955. Estimation of genetic and environmental variability in soybean. J. Agron. 47: 314-318.

Joshi, A.K., Ashwani, K., Sharma, B.K. and Kumar, A. 1998. Evaluation of tomato genotypes for horticultural characteristics. Punjab Veg. Grower 33: 21-22.

Jenkins, J.A. 1948. The origin of cultivated tomato. Econ. Bot. 2: 379.

Kader, A. A. 2008. Flavor quality of grits and vegetables. J. Sci. Food Agric. 88: 1863-1868.

Kader, A.A., Stevens, M.A., Albright-Holton, M., Morris, L.L. and Agazi, M. 1977. Effect of fruit ripeness when picked on flavor and composition in fresh market tomatoes. J. Amer. Soc. Hort. Sci. 102: 724-731.

Krumbein, A. and Auerswald, H. 1998. Characterization of aroma volatiles in tomatoes by sensory analysis. Nahrung. 42 : 395-399.

Lynch, M. and Walsh, B. 1998. Genetics and analysis of quantitative traits. Sinauer Associates Inc. Sunderland, Massachusetts. 823-831.

Miller, J.C., Miller, L., Paterson, A.H., Pineda, O., Roeder, M.S., Wing, R.A., Wu, W. and Young, N. 1991. High density molecular linkage maps of the tomato and potato genomes. Genet. 132: $1141-1160$.

Malundo, T.M.M., Shewfelt, R.L. and Scott, J.W. 1995. Flavor quality of fresh market tomato (Lycopersicon esculentum Mill.) as affected by sugar and acid levels. Postharv. Biol. Technol. 6: $103-110$.

Mohanty, B.K. 2002. Variability, heritability and path coefficient analysis in tomato (Lycopersicon esculentum Mill.). Haryana J. Agric. Sci. 37: (1), 68-71.

Mohanty, B.K. 2003. Studies on variability, heritability, interrelationship and path analysis in tomato. Ann. Agric. Res. 23: (1), 65-69.

McGillivary, J.H. and Clemente, L.J. 1956. Effect of tomato size on solids content. Proc. Amer. Soc. Hort. Sci. 68: 466-469.

Maršić, N.K., Gašperlin, L., Abram, V., Budič, M. and Vidrih, R. 2011. Quality parameters and total phenolic content in tomato fruits regarding cultivar and microclimatic conditions. Turk . J. Agric. For. 35: 185-194.

Mayavel, A., Balakrishnamurthy, G. and Natarajan, S. 2005. Correlation and path analysis in tomato (Lycopersicon esculentum Mill). South Ind. Hort. 53: (1-6), 253-257.

Nakawuka, C.K. and Adipala, E. 1999. A path coefficient analysis of some yield component in cowpea. African crop Sci. J. 4: 327-331.

Osei, M.K., Bonsu, K.O., Agyeman, A. and Choi, H.S. 2014. Genetic diversity of tomato germplasm in Ghana using morphological characters. Int. J. Pl. Soil Sci., 3: (3), 220231.

Reddy, B.R., Reddy, M.P., Reddy, D.S. and Begum, H. 2013. Correlation and path analysis studies foe yield and quality traits in tomato (Solanum lycopersicum L.). IOSR J. Agric. \& Vet. Sci. 4: 56-59.

Reddy, V.V. and Reddy, K.V. 1992. Studies in variability in tomato. South Ind. Hort. 40: 257-260.

Rani, I., Veeraragavathatham, D. and Sanjutha, D. 2008. Studies on Correlation and Path Coefficient analysis on Yield Attributes in Root Knot Nematodes Resistant F1 Hybrids of Tomato. J. Appl. Sci. Res. 4: 287-295.

Rodica, S., Apahidean, S.A., Apahidean, M., Manitiu, D. and Paulette, L. 2008. Yield, Physical and Chemical Characteristics of Greenhouse Tomato Grown on Soil and Organic Substratum. In: 43rd Croatian and 3rd International Symposium on Agriculture. Opatija, Croatia. 439-443.

Shewfelt, R. L. 2000. Consumer friendly specifications for a global markerplace. Food Aust. 52: 311-314.

Shewfelt, R.L. 1993. Measuring quality and maturity, In: R.L. Shewfelt and S.E. Prussia (eds.). Postharvest handling: A systems approach, Academic Press, New York. 100-124.

Stevens, M.A., Kader, A.A., Albright-Holton, M. and Algazi, M. 1977a. Genotypic variation for flavor and composition in fresh market tomatoes. J. Amer. Soc. Hort. Sci. 102: 680689.

Stevens, M.A., Kader, A.A., Albright-Holton, M. 1977b. Intercultivar variation in composition of locular and pericarp portions of fresh market tomatoes. J. Amer. Soc. Hort. Sci. 102: 689692.

Stevens, M. A. 1986. Inheritance of tomato quality components. In: Janick, J. (Ed.), Plant breeding reviews. AVI publishing Co., Westport, CT,:273-311.

Shravan, K., Singh, T., Singh, B. and Singh, J.P. 2004. Studies on heritability and genetic advance in tomato (Lycopersicon esculentum Mill). Prog. Agric. 4: 76-77.

Singh, A.K. and Raj, N. 2004. Variability studies in tomato under cold arid condition of Ladakh. Hort. J. 17: 67-72.

Singh, A.K. 2005. Genetic variability, correlation and path coefficient studies in tomato (Lycopersicon esculentum Mill.) under cold arid region. Prog. Hort. 37:(2), 437-443.

Stevens, M.A. and Rudich, J. 1978. Genetic potential for overcoming physiological limitations on adaptability, yield, and quality in the tomato. Hortsci.13: 673-678.

Tanksley, S.D. 2004. The genetic, developmental and molecular bases of fruit size and shape variation in tomato. The Plant Cell.16: 181-189.

Vickers, A. 1977. Structural and mechanical indicators of flavor quality In: R. Scanlan (Ed.). Flavor quality: objective measurement. ACS Symp. Ser.51. Amer. Chem. Soc., Washington, D.C. 45-50. 